J. Dairy Sci. 92:2653-2661

doi:10.3168/jds.2008-1832

(c) American Dairy Science Association, 2009.

\title{
A longitudinal study on the impact of Johne's disease status on milk production in individual cows
}

\author{
R. L. Smith, ${ }^{\star 1}$ Y. T. Grohn, ${ }^{*}$ A. K. Pradhan,† R. H. Whitlock,‡ J. S. Van Kessel,§ J. M. Smith,\# D. R. Wolfgang, ॥ \\ and Y. H. Schukken† \\ ${ }^{*}$ Section of Epidemiology and \\ †Quality Milk Production Services, Department of Population Medicine and Diagnostic Sciences, College of Veterinary Medicine, \\ Cornell University, Ithaca, NY 14850 \\ $\ddagger$ New Bolton Center, Department of Clinical Studies, School of Veterinary Medicine, University of Pennsylvania, Kennett Square 19348 \\ $\S$ Environmental Microbial Safety Laboratory, ANRI, USDA-ARS, Beltsville, MD 20705 \\ \#Department of Animal Science, University of Vermont, Burlington 05405 \\ ||Department of Veterinary and Biomedical Science, Penn State University, University Park 16802
}

\begin{abstract}
Longitudinal data from 3 commercial dairy herds in the northeast United States were collected from 2004 to 2007. Johne's disease status, as indicated by Mycobacterium avium ssp. paratuberculosis infection levels, was determined through quarterly ELISA serum testing, biannual fecal culture, and culture of tissues at slaughter. Milk production data were collected from the Dairy Herd Improvement Association. The effect of Johne's disease status on milk production was analyzed using a mixed linear model with an autocorrelation random effect structure. Infected animals produced more milk than uninfected cows before they began shedding $M$. avium ssp. paratuberculosis. Cows infected with $M$. avium ssp. paratuberculosis had monthly decreases of 0.05 to $1 \mathrm{~kg}$ in daily milk production relative to uninfected animals, with greater decreases in progressive disease categories. Animals with fecal culture results of $>30 \mathrm{cfu} / \mathrm{g}$ produced approximately $4 \mathrm{~kg}$ less milk per day compared with uninfected cows. These results will be valuable in calculating the economic effect of Johne's disease.
\end{abstract}

Key words: Johne's disease, milk production

\section{INTRODUCTION}

Johne's disease (JD) is a chronic disease of ruminants caused by intestinal infection with the pathogen Mycobacterium avium ssp. paratuberculosis (MAP). The pathogen is pervasive on US dairy farms, with a herd prevalence of approximately $68 \%$ (USDA:APHIS:VS:NAHMS, 2007). Infection with MAP typically occurs in calves (Clarke, 1997), which

Received October 24, 2008.

Accepted January 21, 2009.

${ }^{1}$ Corresponding author: rls57@cornell.edu then enter a latent, nonshedding stage of varying length. This latent stage is followed by a period of low and intermittent shedding of MAP with no obvious clinical symptoms (Whitlock et al., 2000). If allowed to progress, clinically apparent JD may develop, with a high level of MAP shedding (Whitlock et al., 2000).

The most commonly used antemortem tests for identifying MAP shedding are fecal culture and serum ELISA (USDA:APHIS:VS:NAHMS, 2007). Culture techniques are used to determine both the presence and the magnitude of MAP shed in the feces, whereas serum ELISA testing is used to detect an immune response to infection. Both of these tests are sensitive in identifying high-shedding animals, but are much less sensitive for low-shedding animals and often fail to detect latent infections (Eamens et al., 2000; Whitlock et al., 2000). Identification of infected animals is best achieved by repeated sampling (van Schaik et al., 2003). Postmortem culture of tissues, including intestinal epithelium and lymph nodes, can identify infection at any stage, including latency, but the procedure is more sensitive in animals with advanced infections (Huda and Jensen, 2003).

Structured control programs often include immediate culling of high-shedding animals to limit environmental contamination and the transmission of MAP to herdmates, particularly calves. However, a combination of imperfect diagnostic techniques and slow development of clinical symptoms often results in delayed culling or retention of low-shedding animals. Additionally, lowshedding animals are frequently retained due to the high cost of purchasing replacements (Dorshorst et al., 2006).

Johne's disease has been estimated to cost the US dairy industry $\$ 200$ to $\$ 250$ million annually (Ott et al., 1999). The cost of JD is manifested in a variety of ways, but milk production losses are the most insidious. Decreased milk production is believed to occur in 
subclinically and clinically affected animals (Kennedy and Benedictus, 2001), but the precise magnitude of the reduction has not been previously calculated. One study found no significant decrease in 305-d mature equivalent milk production for a small cohort of animals positive for JD by ELISA or fecal culture (Johnson et al., 2001). However, in that study, animals were followed for only 18 mo with tests at the onset and finish of the study. Given the low sensitivity of the diagnostics to low-shedding animals, there was a high probability of misclassification of control animals. Another study noticed a significant decrease in 305-d mature equivalent milk production in fecal culture-positive animals (Wilson et al., 1993). Other studies found that total milk production was decreased over the course of a lactation in fecal culture-positive animals (Raizman et al., 2007) and that ELISA-positive animals had lower 305-d mature equivalent milk production and lifetime milk production (Lombard et al., 2005) compared with test-negative animals. The magnitude of continuous test results from the milk ELISA for MAP antibodies has been found to be significantly related to the shape of the lactation curves (Kudahl et al., 2004).

Each of these 3 studies, however, based their results entirely on a single test per animal and were thus potentially biased by the high probability of false-negative results. False-negative results would misclassify an infected animal as a control and thereby reduce the calculated effect of infection on the outcome of interest. Moreover, an infection status change, for example, from low to high shedding, during the data collection period would not have been taken into account in these studies. Now, more precise estimates of production life can be estimated using test-day milk yield models, which account for random cow effects, repeated observation, and time-dependent covariates indicating disease status (Wilson et al., 2004). Because of the high uncertainty associated with the previous estimates, the reduction of milk production attributed to MAP infection contributes most of the uncertainty in economic models for the cost of JD (Losinger, 2005).

Without accurate milk loss predictors, the economic impact of MAP infection cannot be known with certainty. The objective of this study was therefore to estimate the effect of JD status on individual cow milk production using longitudinal data collected over a 4-yr period from 3 US dairy herds.

\section{MATERIALS AND METHODS}

In 2004, the Regional Dairy Quality Management Alliance identified 1 commercial dairy herd in each of 3 of its member states (New York, Vermont, and Pennsylvania) to serve as a longitudinal study herd. The details of this study have been described previously (Pradhan et al., 2009). Briefly, these 3 herds, which are endemically MAP-infected and consist primarily of Holstein cattle, were visited quarterly to collect samples, and all monthly production, breeding, and health records were obtained through the DHIA. Serum samples were collected quarterly and fecal samples were collected biannually from each adult animal in each herd, and all samples were shipped overnight to The University of Pennsylvania Johne's Laboratory. In addition, samples (including serum, feces, intestinal epithelium, and intestinal lymph nodes) were collected from almost all animals sent to slaughter and most animals that died on-farm during the study period. These samples were refrigerated at the slaughterhouse and shipped within $1 \mathrm{~d}$ of collection. Serum samples were tested by the ParaChek (Prionics USA Inc., La Vista, NE; formerly CSL/Biocor) ELISA for antibody reactions to MAP antigens. Fecal and tissue samples were tested by 4-tube fecal culture for presence of viable MAP organisms (Pradhan et al., 2009). Diagnostic results were reported as positive or negative for ELISA and expressed as colony-forming units per gram for culture.

Johne's disease status (stage of MAP infection) was divided into 4 categories: uninfected, latent, low shedding, and high shedding (defined below). Uninfected animals were defined as animals for which all lifetime diagnostic tests were negative and for which there were at least 2 diagnostic test results reported. Animals with only 1 diagnostic test, if the result was negative, were removed from the analysis due to the lack of diagnostic sensitivity. Animals were assumed infected if at least 1 diagnostic test had a positive result. Johne's disease status was assigned separately for each combination of animal and test day. All milk test days (days on which milk weights were recorded) before the first positive diagnosis were categorized as latent because the animals were assumed to be infected in calfhood. Animals with positive tissue culture results were categorized as latent for all milk test days if no antemortem tests were positive. A cut-off value of $30 \mathrm{cfu} / \mathrm{g}$ was used to separate low- and high-shedding culture results, as representing the midpoint of the moderate-shedding level previously defined by Whitlock et al. (2000). Milk test days after a fecal culture result of at least $30 \mathrm{cfu} / \mathrm{g}$ were categorized as high shedding. All other milk test days in positive animals (falling after a positive diagnosis by ELISA or a fecal culture of $<30 \mathrm{cfu} / \mathrm{g}$ ) were categorized as low shedding. Positive results on ELISA tests are generally considered to follow positive results on fecal culture (van Schaik et al., 2003), so ELISA-positive animals may be categorized as low-shedding rather than latent.

Parity was divided into first and later lactations to account for the different shape of the lactation curve 
in first-parity animals. Calving season was categorized as winter (January to March), spring (April to June), summer (July to September), and fall (October to December). A separate variable was created for milk test-day number (TDnr), indicating the number of milk test days for a given animal since the beginning of the study. It was assumed that 2 milk test days passed between lactations to account for time spent in the dry period, for which the US standard is approximately 60 d. In addition, a variable for the number of milk test days in the current JD status category (MthJ) was created for test-positive animals; MthJ was set equal to zero for uninfected animals. Daily milk production $(\mathrm{kg})$, somatic cell linear score (LS, indicating the log of the SCC), and DIM in the current lactation were used as reported by DHIA, with any recorded non-numeric and zero values for milk production and LS removed from the analysis.

Mixed model analysis was performed using the Mixed procedure in SAS version 9 (SAS Institute Inc., Cary, NC). Wilmink's correction (Wilmink, 1987) was used to model the lactation curve, with an interaction of parity and lactation curve to capture the different shape of the first-lactation curve. Parity, LS, herd, and calving season were included as fixed effects. Cow was included as a random variable, with first-order autoregression for each combination of cow and lactation using TDnr to identify the time lag between observations. The model structure was

$$
\begin{aligned}
\text { Milk }_{i l t} & =\beta_{0}+\beta_{1, p} \text { parity }+\beta_{2} D I M_{i l t} \\
& +\beta_{3} \exp \left[-0.1 \times D I M_{i l t}\right]+\beta_{4} L S_{i l t}+\delta_{1, p} D I M_{i l t} \\
& \times \text { parity }+\delta_{2, p} \exp \left[-0.1 \times D I M_{i l t}\right] \times \text { parity } \\
& +\beta_{5, m} \operatorname{season}+\beta_{6, n} J D_{i l t}+\left(\rho_{i} \varepsilon_{i, t-1}+u_{i l t p m n}\right),
\end{aligned}
$$

where the outcome is daily milk production (in $\mathrm{kg}$ ), $i$ indicates cow, $l$ indicates the present lactation, and $t$ indicates milk test day; $\beta_{1, p}$ is the fixed effect of the dichotomized $p$ th parity $(p=1,>1) ; \beta_{2}$ is the effect of DIM; $\beta_{3}$ is the effect of Wilmink's correction; $\beta_{4}$ is the effect of LS; $\beta_{5, m}$ is the fixed effect of the $m$ th season ( $m=$ winter, spring, summer, fall); and $\beta_{6, n}$ is the fixed effect of the $n$th JD status $(\mathrm{n}=$ uninfected, latent, low-shedding, high-shedding). The interaction coefficient $\delta_{1, p}$ is the effect of the interaction between DIM and the $p$ th parity, and the interaction coefficient $\delta_{2, p}$ is the effect of the interaction between Wilmink's correction and the $p$ th parity. The term $\rho_{i} \varepsilon_{i, t-1}$ provides the first-order autoregression between milk test days in individual cows; and $u_{i l t p m n}$ is the error term for each test date. Differences in milk production were tested between each level of JD status using a standard Ftest.

A subsequent model was built to take into account the monthly decrease in milk production within each JD status category, with the structure

$$
\begin{aligned}
\text { Milk }_{i l t} & =\beta_{0}+\beta_{1, p} \text { parity }+\beta_{2} D I M_{i l t} \\
& +\beta_{3} \exp \left[-0.1 \times D I M_{i l t}\right]+\beta_{4} L S_{i l t}+\delta_{1, p} D I M_{i l t} \\
& \times \text { parity }+\delta_{2, p} \exp \left[-0.1 \times D I M_{i l t}\right] \times \text { parity } \\
& +\beta_{5, m} \text { season }+\beta_{6, n} J D_{i l t}+\beta_{7} M t h J_{i l t}+\beta_{8, n} J D_{i l t} \\
& \times M \text { th }_{i l t}+\left(\rho_{i} \varepsilon_{i, t-1}+u_{i l t p m n}\right),
\end{aligned}
$$

where most parameters are as defined in equation [1] and $\beta_{7}$ is the linear effect of MthJ, $\beta_{8, n}$ is the fixed effect of the interaction between MthJ and the $n$th JD status, and MthJ is a measure of time spent at the current JD status (described above). This model is hereafter defined as the time-based model. All effects were considered significant at $\alpha=0.05$ level.

\section{RESULTS}

A description of the data available for analysis is included in Table 1 . There were 24,474 observations available for analysis, representing 1,332 individual cows and 2,713 individual lactations (934 first lactations) for the period from January 2003 to December 2007. No fecal culture results had been reported for 2007 at the time of analysis. In total, 2,775 observations, approximately $10 \%$ of all observations, were deleted because they lacked sufficient test results (at least 1 positive or 2 negative tests), appropriate milk weights, or LS (numeric, nonzero values).

Convergence criteria were met for all models. The results of the 2 models used are presented in Table 2 . Classes not included in the variable list were considered baseline values and are included in the intercept. Tests of significance between JD status levels based on each of the models are shown in Table 3. All meaningful comparisons are included. Inclusion of a linear variable for the colony-forming units per gram recorded in the most recent positive fecal culture resulted in a small, nonsignificant parameter estimate, and was therefore removed from the model.

Several cows had recorded lactation lengths that were much longer than typical; although the expected lactation length is approximately $1 \mathrm{yr}, 2,828$ observations were more than 365 DIM and 104 observations were more than 730 DIM. This is likely because of a failure to report late-term abortions as the onset of new lactations, as many of these lactation curves resembled 
2 contiguous lactation curves. If all observations with DIM $>390 \mathrm{~d}$ are removed from the analysis, the results of the categorical model are not changed significantly but $M t h J$ time in the time-based model becomes borderline significant (data not shown).

A graph of average predicted milk production compared with average observed milk production over the first 5 lactations in herd $\mathrm{A}$ is shown in Figure 1 to demonstrate that the model adequately predicts the shape of the lactation curve for the different parities; because of the similarities between cows in the older $(\geq 2)$ parities in the analysis, only the first 2 parities were figured. Figure 2 shows the predicted lactation curves for different levels of infection for an average cow in herd A in the third lactation. Predicted values in both curves are generated with the time-based model (equation [2]).

\section{DISCUSSION}

The model presented is the first assessment of the effect of JD on milk production in individual animals over a period of several years. The results may be somewhat limited by the lack of large numbers of test-day results for high-shedding animals, as few animals are retained in the herds when they are known to be shedding large quantities of MAP. Producers in the study herds were made aware of test results and were encouraged by their veterinarian, in association with the research team, to cull these animals immediately. The results may also be biased by the lack of diagnostic results for approximately $10 \%$ of animals in the study, which may have caused the loss of data on animals (especially low-producing animals) culled before diagnosis was possible. However, the latter would have biased the results observed toward nonsignificance. In addition, no fecal culture results were available for 2007, which may have misclassified animals as uninfected or low shedding. This misclassification, however, would be uninformative (assuming no correlation between milk production and presence in the herd in 2007), and should not bias the results.

All possible confounding variables included in the model (parity, DIM, LS, and calving season) were significant, with robust parameter values similar to those observed in other models of milk production (Macciotta et al., 2005), implying a large degree of stability in the analysis.

An interaction term was included to account for the unique shape of the lactation curve in first-lactation cows. Cows in their first lactation could have been modeled separately from multiparous animals. However, with the long latent period in JD, the majority of MAP-infected first-lactation cows would be latent

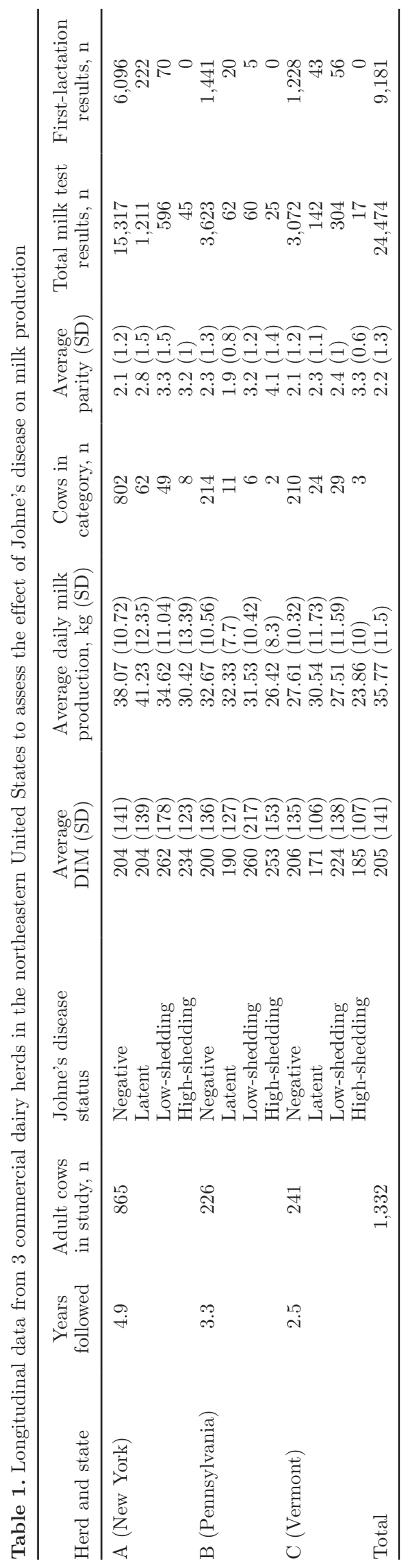


Table 2. Results of 2 linear mixed models for daily milk production $(\mathrm{kg})^{1}$

\begin{tabular}{|c|c|c|c|c|c|}
\hline Variable & Class & \multicolumn{2}{|c|}{ Categorical } & \multicolumn{2}{|c|}{ Time-based } \\
\hline Intercept & & 33.38 & $70.79(<0.001)$ & 33.29 & $69.70(<0.001)$ \\
\hline DIM & & -0.03 & $-25.06(<0.001)$ & -0.03 & $-25.00(<0.001)$ \\
\hline $\mathrm{e}^{-0.1 \times \mathrm{DIM}}$ & & -24.45 & $-28.28(<0.001)$ & -24.45 & $-28.30(<0.001)$ \\
\hline DIM $\times$ parity (base is first lactation) & $>1$ lactation & -0.04 & $-26.64(<0.001)$ & -0.04 & $-26.65(<0.001)$ \\
\hline \multirow[t]{3}{*}{ Calving season (base is winter) } & Fall & -0.48 & $-1.49(0.181)$ & -0.50 & $-1.53(0.170)$ \\
\hline & Spring & -0.43 & $-1.26(0.247)$ & -0.38 & $-1.08(0.314)$ \\
\hline & Summer & -1.44 & $-4.32(0.004)$ & -1.41 & $-4.19(0.004)$ \\
\hline \multirow[t]{3}{*}{ JD status (base is uninfected) } & Latent & 2.30 & $5.06(<0.001)$ & 3.15 & $4.73(<0.001)$ \\
\hline & Low-shedding & 0.20 & $0.37(0.709)$ & 0.86 & $1.20(0.234)$ \\
\hline & High-shedding & -3.70 & $-1.94(0.056)$ & 0.84 & $0.33(0.744)$ \\
\hline JD time $\times$ JD status (base is high shedding) & & & & -1.12 & $-2.54(0.011)$ \\
\hline
\end{tabular}

${ }^{1}$ In the first model, only the categorical Johne's disease (JD) status of the animal at the time of the milk test is considered. In the second model, the number of months spent in the JD status category is added to the model (JD time $=0$ for uninfected animals). Herd was included as a fixed effect in the model, but is not reported here.

and the latent first-lactation animals would represent a small proportion of the total MAP-infected group. This would decrease the power of our analyses; therefore, it was more valuable to combine all data in one model. As Figure 1 demonstrates, the final model was well able to predict the shape of the first lactation curve.

Johne's disease status was found to have a significant effect on milk production, and this effect was not uniform across JD status categories. This confirms the importance of separating latent, low-shedding, and high-shedding animals in the analysis, as well as the importance of the category definitions. However, as seen in Tables 2 and 3, the effect is not always significant between groups. The difference between latent and negative or latent and low-shedding animals is relatively large and statistically significant. The difference between negative and low-shedding animals is small and nonsignificant. Our observation that latent animals show an approximately $2 \mathrm{~kg}$ higher daily milk production compared with negative animals is not fully understood. However, this observation is very similar to what is observed in other infectious diseases in dairy cows. Cows with higher milk production are more susceptible to clinical mastitis (Bar et al., 2007), a relationship partly explained by a positive genetic correlation between milk production and mastitis. Similarly, in our data, cows that eventually will show low and high shedding of MAP are out-producing MAP-negative animals in the herd. Following the same argument, we speculate that there may be a genetic component to JD suscep-

Table 3. Comparison tests for the effect of Johne's disease (JD) status levels on daily milk production ${ }^{1}$

\begin{tabular}{|c|c|c|c|c|c|c|}
\hline Comparison & \multicolumn{3}{|c|}{ Categorical } & \multicolumn{3}{|c|}{ Time-based } \\
\hline Latent vs. negative & +2.3 & 5.06 & $<0.001$ & $\begin{array}{l}+3.15 \\
-0.04 \times M t h J\end{array}$ & 4.73 & $<0.001$ \\
\hline Latent vs. low-shedding & +2.1 & 13.40 & $<0.001$ & $\begin{array}{l}+2.29 \\
+0.03 \times M t h J\end{array}$ & 0.15 & 0.695 \\
\hline Latent vs. high-shedding & +6.0 & 9.82 & 0.003 & $\begin{array}{l}+2.31 \\
+2.2 \times M t h J\end{array}$ & 5.94 & 0.015 \\
\hline Low-shedding vs. high-shedding & +3.9 & 3.99 & 0.050 & $\begin{array}{l}+0.02 \\
+2.17 \times M t h J\end{array}$ & 5.59 & 0.018 \\
\hline High-shedding vs. negative & -3.7 & -1.94 & 0.056 & $\begin{array}{l}+0.84 \\
-1.12 \times M t h J\end{array}$ & -2.54 & 0.011 \\
\hline
\end{tabular}

${ }^{1}$ The categorical model only considers the JD status at the time of the milk test. The time-based model also considers the effect of the number of months the animal has spent in the JD status category (MthJ). 


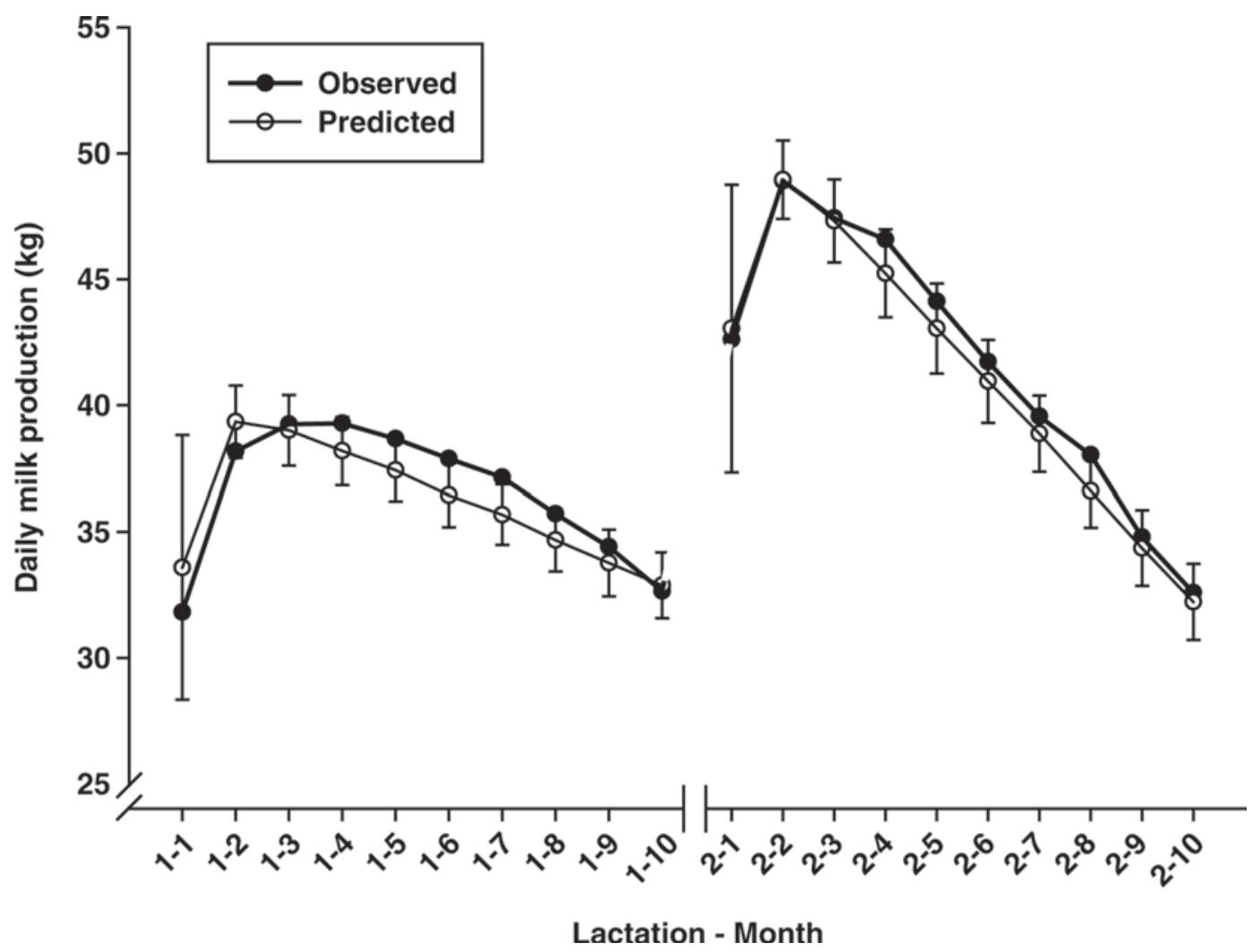

Figure 1. Average predicted and observed lactation curves for uninfected cows in herd A, starting in first parity. The predicted values come from the full model, as detailed in Table 2 .

tibility, with a possible positive genetic correlation to greater milk production. This was not observed in one study examining a genetic link between ELISA values and milk production, but that study was not able to separate latent and uninfected animals in their analysis (Mortensen et al., 2004). In terms of loss of potential milk production, we would argue that MAP-infected latent cows have a production potential that is $2.3 \mathrm{~kg}$ higher than uninfected herdmates. It is possible that low-producing latent cows were culled before infection became detectable, but the use of tissue culture at slaughter should have minimized that bias. The decrease in daily milk production due to low shedding $(2.1 \mathrm{~kg})$ and high shedding $(6.0 \mathrm{~kg})$ of MAP would have to be accounted for in a complete economic analysis of JD. These are useful numbers for the producer to have when making economic decisions. Culling of lowshedding or high-shedding animals may be based solely on the negative effect of JD on milk production, but should also take into account the contribution of shedders to MAP infection spread within the herd and other potential negative economic effects, such as delayed reproduction. In the study herds, culling decisions were made with knowledge of MAP diagnostic results, but cattle were culled more often for low production and reproductive problems than for JD (data not shown).

In terms of this analysis, these results explain the previously observed lack of a significant difference in milk production between MAP-uninfected and MAPinfected animals: the higher milk production in latently MAP-infected animals balances out the lower milk production in high-shedding animals (Johnson et al., 2001). Additionally, the stress associated with failing to meet the nutritional requirements of high milk production could increase the probability of shedding MAP in the future.

Considering only categorical JD status, there is a significant decrease in milk production when animals move between subsequent levels of JD status. This decrease is especially pronounced between low- and highshedding animals. These results are not unexpected: high-shedding animals are more likely to be clinically affected by JD, with decreased performance linked to decreased intestinal absorptive capabilities (JohnsonIfearulundu and Kaneene, 1997).

When a variable is added to the time-based model for time spent in any given JD status category, the progression of JD leads to an increase in milk production 


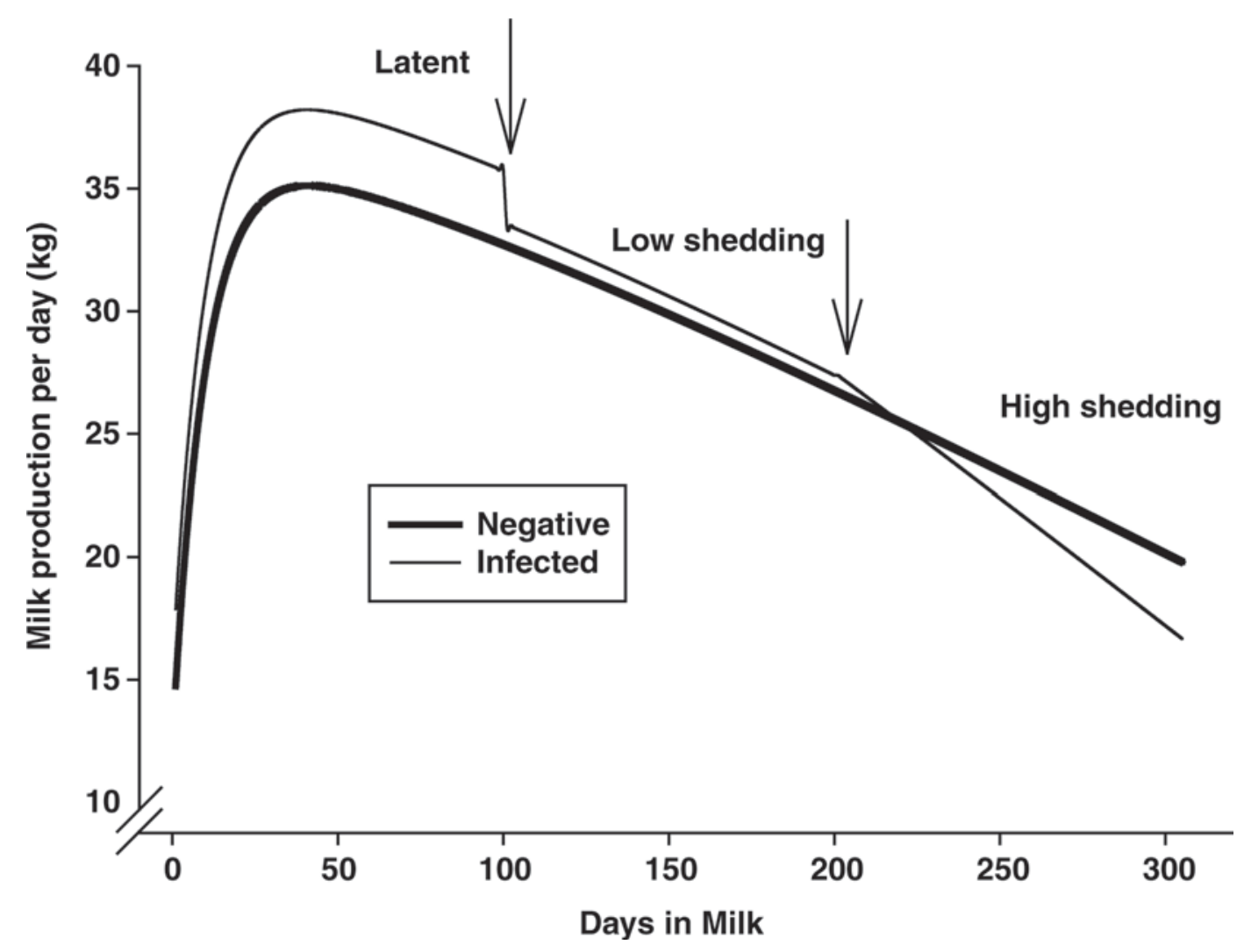

Figure 2. Predicted milk yield $(\mathrm{kg})$ for an average cow in herd A in the third lactation, comparing an uninfected animal with an animal with changing infection status levels (at 100 and 200 DIM, indicated by arrows). Changes in infection status levels were simulated for demonstration purposes.

loss over time. Although latent animals produce more milk than uninfected animals, that difference decreases over time in the latent infection state. When an animal starts shedding low levels of MAP, the model predicts an initial milk production that is slightly higher than that of uninfected herdmates, but there is a greater rate of decrease in milk production compared with the latently infected animals. Finally, animals in the high-shedding category have a meaningfully lower milk production than uninfected herdmates, with large decreases in production over time when remaining in the herd.

Figure 2 shows the predicted lactation curves for an average animal in herd A compared across JD status categories. Greater milk yield is evident during latency compared with uninfected herdmates, but the discrepancy in yield decreases as the disease progresses over time. This MAP-induced decrease in milk production is supported by the clinical progression of JD. As the organism invades the intestinal epithelium and begins to affect nutrient absorption, feed efficiency decreases and milk production is negatively affected. This is also consistent with the findings of Kudahl et al. (2004), who demonstrated that an increase in ELISA positivity (optical density values) caused depressed lactation curves with more negative (decreasing) milk production slopes in late lactation. The magnitude of this effect was reported to have increased with parity (Kudahl et al., 2004). An advantage of time-based models such as the one described here (equation [2]) is the potential to study the continuous nature of biological processes such as disease progression and milk production. Culling is a daily or weekly decision, and by modeling the biological process as closely as possible, the optimal time for culling any individual JD-positive animal can be narrowed to a specific month after testing results.

The main disadvantage of these models is the quality of data gathered from the available diagnostics. Sensitivity is low, especially with fecal culture, and recent studies have cast doubt on the specificity of fecal culture (Whitlock et al., 2000). Some of the animals considered JD-negative in these data might well have been latently infected or even intermittently shedding low quantities of MAP. Some of the low-shedding (or ELISA-positive only) animals may be experiencing "passive infections," in which they are not truly shedding MAP, but are 
simply latently infected animals serving as passive vectors for MAP organisms present in their environment (Whitlock et al., 2008).

It was assumed that all ELISA-positive animals were low shedding unless a high-positive fecal culture existed. This should be an acceptable assumption, as ELISA results indicative of heavy shedding have been observed to follow the corresponding fecal culture results (van Schaik et al., 2003). However, fecal culture frequency was lower than that of ELISA tests (biannual compared with quarterly), so some animals may have been misclassified as low shedding for several months after high shedding began. In this case, the milk test results (with a monthly frequency) would be misclassified. It would be possible to assume a backdated positive status; for example, a date between low-shedding and high-shedding results. However, producers will rarely perform fecal culture more than once a year, so MAP shedding results are generally available only on an annual basis.

Additionally, it was assumed that all MAP-infected animals were infected as calves. This allowed animals to be categorized as latent at all test days before their first positive diagnosis. The idea of calfhood infection or, at the very latest, infection in the first year of life, is supported by a recent review article (Begg and Whittington, 2008), so this assumption was considered reasonable.

This analysis provides strong support that JD status affects milk production in all infected animals, with increasing losses in milk production as disease progresses. The above-average milk production of animals later affected by JD further highlights the important loss of milk production in high potential animals. These results will be useful in making culling decisions on an individual-animal, economic level, especially as animals shedding MAP also spread the infection through environmental contamination.

\section{ACKNOWLEDGMENTS}

The USDA Cooperative State Research, Education and Extension Service (Washington, DC) Award Number 2008-35204-04627 provided funding for this study, as did the USDA-Agricultural Research Service (Agreements. 58-1265-3-155, 58-1265-3-156, 58-1265-3158, and 58-1265-4-020) for the Regional Dairy Quality Management Alliance and the Johne's Disease Integrated Program (JDIP, USDA contract 45105).

\section{REFERENCES}

Bar, D., Y. T. Grohn, G. Bennett, R. N. Gonzalez, J. A. Hertl, H. F. Schulte, L. W. Tauer, F. L. Welcome, and Y. H. Schukken. 2007.
Effect of repeated episodes of generic clinical mastitis on milk yield in dairy cows. J. Dairy Sci. 90:4643-4663.

Begg, D. J., and R. J. Whittington. 2008. Experimental animal infection models for Johne's disease, an infectious enteropathy caused by Mycobacterium avium subsp. paratuberculosis. Vet. J. 176:129-145.

Clarke, C. J. 1997. The pathology and pathogenesis of paratuberculosis in ruminants and other species. J. Comp. Pathol. 116:217-261.

Dorshorst, N. C., M. T. Collins, and J. E. Lombard. 2006. Decision analysis model for paratuberculosis control in commercial dairy herds. Prev. Vet. Med. 75:92-122.

Eamens, G. J., R. J. Whittington, I. B. Marsh, M. J. Turner, V. Saunders, P. D. Kemsley, and D. Rayward. 2000. Comparative sensitivity of various faecal culture methods and ELISA in dairy cattle herds with endemic Johne's disease. Vet. Microbiol. 77:357367.

Huda, A., and H. E. Jensen. 2003. Comparison of histopathology, cultivation of tissues and rectal contents, and interferon-gamma and serum antibody responses for the diagnosis of bovine paratuberculosis. J. Comp. Pathol. 129:259-267.

Johnson, Y. J., J. B. Kaneene, J. C. Gardiner, J. W. Lloyd, D. J. Sprecher, and P. H. Coe. 2001. The effect of subclinical Mycobacterium paratuberculosis infection on milk production in Michigan dairy cows. J. Dairy Sci. 84:2188-2194.

Johnson-Ifearulundu, Y. J., and J. B. Kaneene. 1997. Epidemiology and economic impact of subclinical Johne's disease: A review. Vet. Bull. 67:437-447.

Kennedy, D., and G. Benedictus. 2001. Control of Mycobacterium avium subsp. paratuberculosis infection in agricultural species. Rev. Sci. Tech. 20:151-179.

Kudahl, A., S. S. Nielsen, and J. T. Sorensen. 2004. Relationship between antibodies against Mycobacterium avium subsp. paratuberculosis in milk and shape of lactation curves. Prev. Vet. Med. 62:119-134.

Lombard, J. E., F. B. Garry, B. J. McCluskey, and B. A. Wagner. 2005. Risk of removal and effects on milk production associated with paratuberculosis status in dairy cows. J. Am. Vet. Med. Assoc. 227:1975-1981.

Losinger, W. C. 2005. Economic impact of reduced milk production associated with Johne's disease on dairy operations in the USA. J. Dairy Res. 72:425-432.

Macciotta, N. P. P., D. Vicario, and A. Cappio-Borlino. 2005. Detection of different shapes of lactation curve for milk yield in dairy cattle by empirical mathematical models. J. Dairy Sci. 88:1178-1191.

Mortensen, H., S. S. Nielsen, and P. Berg. 2004. Genetic variation and heritability of the antibody response to Mycobacterium avium subspecies paratuberculosis in Danish Holstein cows. J. Dairy Sci. 87:2108-2113

Ott, S. L., S. J. Wells, and B. A. Wagner. 1999. Herd-level economic losses associated with Johne's disease on US dairy operations. Prev. Vet. Med. 40:179-192.

Pradhan, A., J. S. Van Kessel, J. S. Karns, D. R. Wolfgang, E Hovingh, K. A. Nelen, J. M. Smith, R. H. Whitlock, T. Fyock, S. Ladely, P. J. Fedorka-Cray, and Y. H. Schukken. 2009. Dynamics of endemic infectious diseases of animal and human importance on three dairy herds in the northeastern United States. J. Dairy Sci. 92:1811-1825.

Raizman, E. A., J. Fetrow, S. J. Wells, S. M. Godden, M. J. Oakes, and G. Vazquez. 2007. The association between Mycobacterium avium subsp. paratuberculosis fecal shedding or clinical Johne's disease and lactation performance on two Minnesota, USA dairy farms. Prev. Vet. Med. 78:179-195.

USDA:APHIS:VS:NAHMS. 2007. NAHMS 2007: Johne's Disease on U.S. Dairy Operations. Report \#N521.0408. UDSA:APHIS, Fort Collins, CO.

van Schaik, G., C. R. Rossiter, S. M. Stehman, S. J. Shin, and Y. H. Schukken. 2003. Longitudinal study to investigate variation in results of repeated ELISA and culture of fecal samples for Mycobacterium avium subsp paratuberculosis in commercial dairy herds. Am. J. Vet. Res. 64:479-484. 
Whitlock, R., R. W. Sweeney, J. Smith, J. S. Van Kessel, E. Hovingh, J. Karns, D. Wolfgang, T. Fyock, S. McAdams, and Y. H. Schukken. 2008. Cattle shedding MAP: A new paradigm, passive shedding or active shedding? Page 12 in Proc. 4th Ann. Conf. Johne's Disease Integrated Program. USDA-CSREES-NRI-CAP, East Lansing, MI.

Whitlock, R. H., S. J. Wells, R. W. Sweeney, and J. Van Tiem. 2000. ELISA and fecal culture for paratuberculosis (Johne's disease): Sensitivity and specificity of each method. Vet. Microbiol. $77: 387-398$.

Wilmink, J. B. M. 1987. Adjustment of test-day milk, fat, and protein yield for age, season and stage of lactation. Livest. Prod. Sci. $16: 335-348$.
Wilson, D. J., R. N. Gonzalez, J. A. Hertl, H. F. Schulte, G. J. Bennett, Y. H. Schukken, and Y. T. Grohn. 2004. Effect of clinical mastitis on the lactation curve: A mixed model estimation using daily milk weights. J. Dairy Sci. 87:2073-2084.

Wilson, D. J., C. A. Rossiter, H. R. Han, and P. M. Sears. 1993. Association of Mycobacterium paratuberculosis infection with reduced mastitis, but with decreased milk production and increased cull rate in clinically normal dairy cows. Am. J. Vet. Res. 54:1851-1857. 